\title{
Social Life Factors Affecting Stillbirth, and Infant and Adult-Disease Mortality
}

\author{
Shunichi Araki and Katsuyuki Murata \\ Department of Public Health and Hygiene, Medical College \\ of Oita, Oita 879-56
}

Araki, S. and Murata, K. Social Life Factors Affecting Stillbirth, and Infant and Adult-Disease Mortality. Tohoku J. exp. Med., 1986, 149 (2), 213-219 The effects of a wide variety of social life factors on stillbirth rate as well as infant and adult-disease mortality rates in 46 prefectures in Japan were analyzed by stepwise regression analysis twice at a 5-year interval. Adult-disease mortality was calculated by summing age-adjusted mortality from five major causes of death. The results indicated that rural residence was the key factor affecting infant and adult-disease mortality ; low income, together with social mobility, was the principal factor for stillbirth. International differences in the effects of urbanisation on these mortality rates are discussed in the light of these findings. —— stillbirth ; infant mortality; adult-disease mortality ; rural residence ; low income

Our previous study indicated that rural residence was the key factor affecting the mortality of total Japanese population, and that low income was another important factor for the mortality of the male population (Araki and Murata 1986b). These factors also significantly affected the mortality of some working populations and suicide mortality in Japan (Araki and Murata 1984a, b, 1985, 1986a).

In Western countries, however, it appears that the effects of urbanisation on mortality are reverse ; the risk of death is higher in urban residence. For example, in Great Britain, mortality rates are highest in the conurbations and decrease through large and medium sized towns to its lowest level in small towns and rural areas (McKeown and Lowe 1974). Similarly, in the United States, metropolitan areas tend to have higher mortality rates than nonmetropolitan areas (Sauer 1980).

In this study, we apply our previous method to the analyses of stillbirth and infant and adult-disease mortality. The effects of a wide variety of social life factors on these mortality rates are analyzed by stepwise regression analysis after classification of the factors by factor analysis in 46 prefectures in Japan.

\section{Materials and Methods}

The study is based on the following data in all but Okinawa prefectures (i.e., 46 prefectures) in Japan: (1) stillbirth rate and infant and adult-disease mortality rates

Received February 10, 1986 ; accepted for publication May 6, 1986. 
(Japan Ministry of Health and Welfare 1974, 1978; Japan Prime Minister's Office 1977), and (2) 20 variables of social life factors (Japan Prime Minister's Office 1977). Stillbirth rate was defined as the number of spontaneous and artificial stillbirths per 1,000 total births (inclusive of foetal deaths) over 12 weeks gestation; infant mortality rate was the number of infants who died under one year of age per 1,000 live births. Adult-disease mortality rate, defined by Japan Ministry of Health and Welfare as the sum of mortality rates for malignant neoplasms (ICD number 140-209), diabetes mellitus (250), heart disease (393-398, 410-429), hypertensive disease (400-404) and cerebrovascular disease (430-438) (Japan Prime Minister's Office 1977), was calculated by summing the age-adjusted mortality rates (adjusted to total Japanese male or female population in 1960) for these five causes of death (Japan Ministry of Health and Welfare 1974, 1978). These data were available for 1970 and for 1975. The analysis was conducted using Nippon Electric Corporation PC-9801 computer (Tokyo).

A total of 22 social life indicators (Table 1 and Fig. 1), including 20 variables for this study, have been selected from the Japanese edition of the System of Statistical Indicators on Life [one or more indicators from each of the 13 major categories of social life (a total of 227 indicators, Japan Prime Minister's Office 1977)] ; the all 22 indicators have been classified into eight groups of factors for the year 1970 and seven groups for 1975 as extracted by factor analysis in our previous study (Araki and Murata 1985, 1986a). The first factor identified in both the 1970 and 1975 study was rural or urban residence; the second was low income and unemployment; the third was prefectural age distribution; and, in 1970, the fourth was related to home help for the elderly and, in 1975, to social mobility. The first group of social life indicators classified on the basis of these factors, comprising Indicator 9 (Table 1), represented rural residence in both 1970 and 1975 ; the second group, comprising Indicator 1, represented urban residence; the third was low
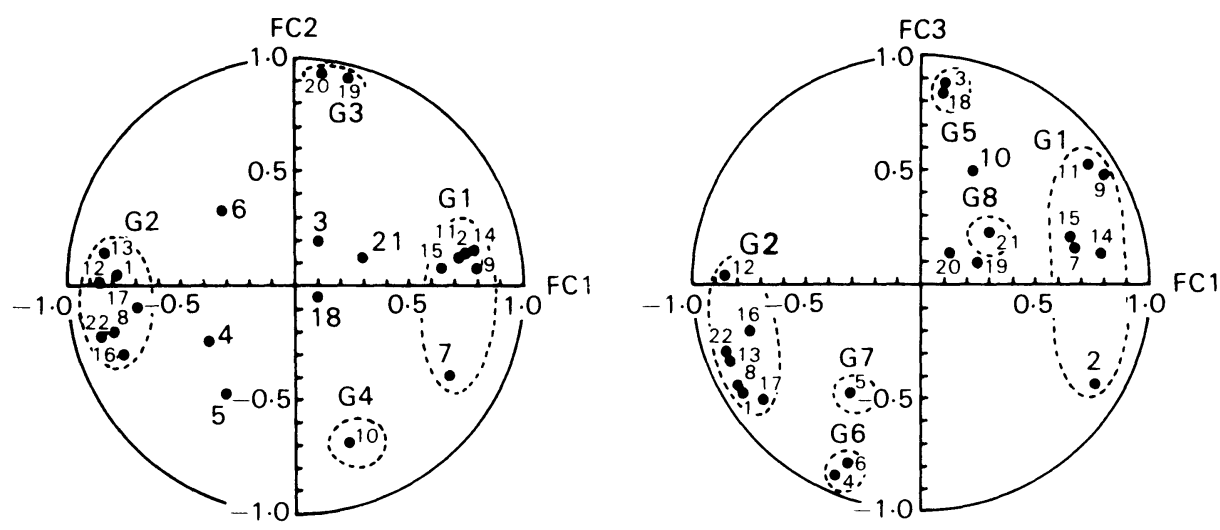

Fig. 1. Eight groups of factors classified from 22 social life indicators in 46 Japanese prefectures by factor analysis in 1970 (Araki and Murata 1985, 1986a). FC 1, 2 and $3=$ the first, second and third factor, respectively, extracted by factor analysis. The numbers of the indicators are same as in Table 1. The indicators have been classified into 8 groups (G1 to G8) according as the factor loadings were more than 0.5 or less than -0.5 on the basis of the 4 factors extracted: $\mathrm{G} 1=$ rural residence, $\mathrm{G} 2=$ urban residence, $\mathrm{G} 3=$ low income, $\mathrm{G} 4=$ high employment, $\mathrm{G} 5=$ old age group, $\mathrm{G} 6=$ young age group, $\mathrm{G} 7=$ social mobility, and $\mathrm{G} 8=$ social isolation of elderly. Eigen values were $11.5,3.2,2.6$ and 0.9 for the first, second, third and fourth factor, respectively ; cumulative proportions were $0.52,0.67,0.79$ and 0.83 up to the first, second, third and fourth factor, respectively. 
TABLE 1. Twenty-two social life indicators in 46 prefectures for 1970 and 1975 classified by factor analysis

Natural environment:

1 Population density (G2)*

Population and household:

2 Young persons aged 14 and less, \% (G1 for 1970, G6 for 1975)

3 Persons aged 65 and over, \% (G5)

4 Natural growth in population (G6)

5 Population growth by social mobility (G7)

6 Nuclear family, \% (G6)

7 Persons per household (G1 for 1970, G4 for 1975)

Economic basis and labor:

8 Revenue of prefecture per population (G2)

9 Proportion of farmers, fishermen and forest workers (G1)

10 Employment rate per population aged 15 and over (G4)

11 Persons working away from home per population (G1)

School education :

12 Persons entering courses of higher education, \% (G2)

Medical service :

13 Hospitals and clinics per residential area (G2)

Health and safety $: \dagger$

14 Standardized mortality rate (G1)

15 Infant mortality (G1)

Household economy :

16 Savings per household (G2)

Living environment:

17 Domestic water supply, \% (G2)

18 Retail shops per population (G5)

Social security and finance:

19 Persons receiving family income supplement, \% (G3)

20 Expense of family income supplement per population (G3)

21 Home helpers for elderly aged 65 and over (G8 for 1970, G1 for 1975)

Culture :

22 Newspaper circulation per population (G2)

* Group numbers of factors are shown in parentheses (Araki and Murata 1985, 1986a).

$\dagger$ These two indicators are not used for stepwise regression analysis in this study.

income group; the fourth was high employment; the fifth was old age; the sixth was young age; the seventh was social mobility (population growth); and Indicator 21 (home help for the elderly) was the eighth group in 1970, but moved into the first place in 1975 .

Reflecting the post-World War II rapid decline in mortality rate in Japan both in men and women, the age-adjusted mortality in men was 1,166, 823, 654 and 470 deaths per 100,000 population in $1950,1960,1970$ and 1980 , respectively, according to our calculation based on the vital statistics data (Japan Ministry of Health and Welfare 1984). 


\section{RESUlts}

The results of stepwise regression analysis are shown in Table 2. In both 1970 and 1975, infant mortality rate was significantly related to rural residence factor (Indicators 2 and 9). Similarly, in the two years, adult-disease mortality rate was inversely related to urban residence factor (Indicator 12) in both men and women; the rate was inversely related to old age group factor (Indicator 3) in men. Stillbirth rate was positively related to low income factor (Indicator 19), and was inversely related to social mobility (population growth) factor (Indicator

TABLE 2. Social life indicators (group number in parentheses) affecting infant and adult-disease mortality and stillbirth rates in 46 prefectures selected by stepwise regression analysis

\begin{tabular}{|c|c|c|c|c|c|}
\hline & \multicolumn{3}{|c|}{1970} & \multicolumn{2}{|r|}{1975} \\
\hline & $\mathrm{R} \dagger$ & $\begin{array}{l}\text { Indicator } \\
\text { (Group, }\end{array}$ & $\begin{array}{l}\text { rs selected } \\
t \text { value } \ddagger\end{array}$ & $\mathrm{R} \dagger$ & $\begin{array}{l}\text { Indicators selected } \\
\text { (Group, } t \text { value) } \ddagger\end{array}$ \\
\hline Infant mortality & 0.841 & $\begin{aligned} 2 & (\mathrm{G} 1, \\
21 & (\mathrm{G} 8, \\
9 & (\mathrm{G} 1, \\
18 & (\mathrm{G} 5,\end{aligned}$ & $\begin{array}{l}3.305) \\
2.669) \\
2.493) \\
2.220)\end{array}$ & 0.564 & $9(\mathrm{Gl}, \quad 4.534)$ \\
\hline \multicolumn{6}{|c|}{ Adult-disease mortality: } \\
\hline Men & 0.890 & $\begin{aligned} 3 & (\mathrm{G} 5, \\
4 & (\mathrm{G} 6, \\
12 & (\mathrm{G} 2, \\
5 & (\mathrm{G} 7,\end{aligned}$ & $\begin{array}{r}-6.857) \\
-5.908) \\
-4.135) \\
2.863)\end{array}$ & 0.848 & $\begin{array}{rrr}3 & (\mathrm{G} 5, & -4.835) \\
9 & (\mathrm{G} 1, & 3.009) \\
18 & (\mathrm{G} 5, & 2.646) \\
12 & (\mathrm{G} 2, & -2.282)\end{array}$ \\
\hline Women & 0.757 & $\begin{aligned} 12 & (\mathrm{G} 2, \\
7 & (\mathrm{G} 1, \\
8 & (\mathrm{G} 2,\end{aligned}$ & $\begin{array}{r}-5.169) \\
2.577) \\
2.043)\end{array}$ & 0.644 & $\begin{array}{rll}3 & (\mathrm{G} 5, & -3.409) \\
12 & (\mathrm{G} 2, & -3.341) \\
6 & (\mathrm{G} 6, & -2.582)\end{array}$ \\
\hline Stillbirth rate & 0.772 & $\begin{aligned} 5 & (\mathrm{G} 7, \\
19 & (\mathrm{G} 3\end{aligned}$ & $\begin{array}{r}-4.184) \\
2.913)\end{array}$ & 0.837 & $\begin{array}{rlr}19 & (\mathrm{G} 3, & 4.955) \\
16 & (\mathrm{G} 2, & -3.450) \\
5 & (\mathrm{G} 7, & -2.437)\end{array}$ \\
\hline
\end{tabular}

$\dagger$ Multiple correlation coefficient.

$\ddagger$ The number of social life indicators and groups as in Table 1 and Fig. 1 (the significance level for entering and removing variables $p<0.05$ ).

TABLE 3. Differences in infant and adult-disease mortality and stillbirth rates in 46 prefectures in Japan 1970 and 1975 (paired-sample t-test)

\begin{tabular}{|c|c|c|c|}
\hline & 1970 & 1975 & $p$ values \\
\hline Infant mortality (per $10^{3}$ live births) & $14.2(2.0)$ & $10.5(1.4)$ & $<0.01$ \\
\hline \multicolumn{4}{|c|}{ Adult-disease mortality (per $10^{5}$ population) : } \\
\hline Men & $371 \quad(35)$ & $322 \quad(21)$ & $<0.01$ \\
\hline Women & $312 \quad(23)$ & $276 \quad(16)$ & $<0.01$ \\
\hline Stillbirth rate (per $10^{3}$ total births) & $67.9(13.1)$ & $52.2(9.2)$ & $<0.01$ \\
\hline
\end{tabular}

Mean values and S.D. (in parentheses) are given. 

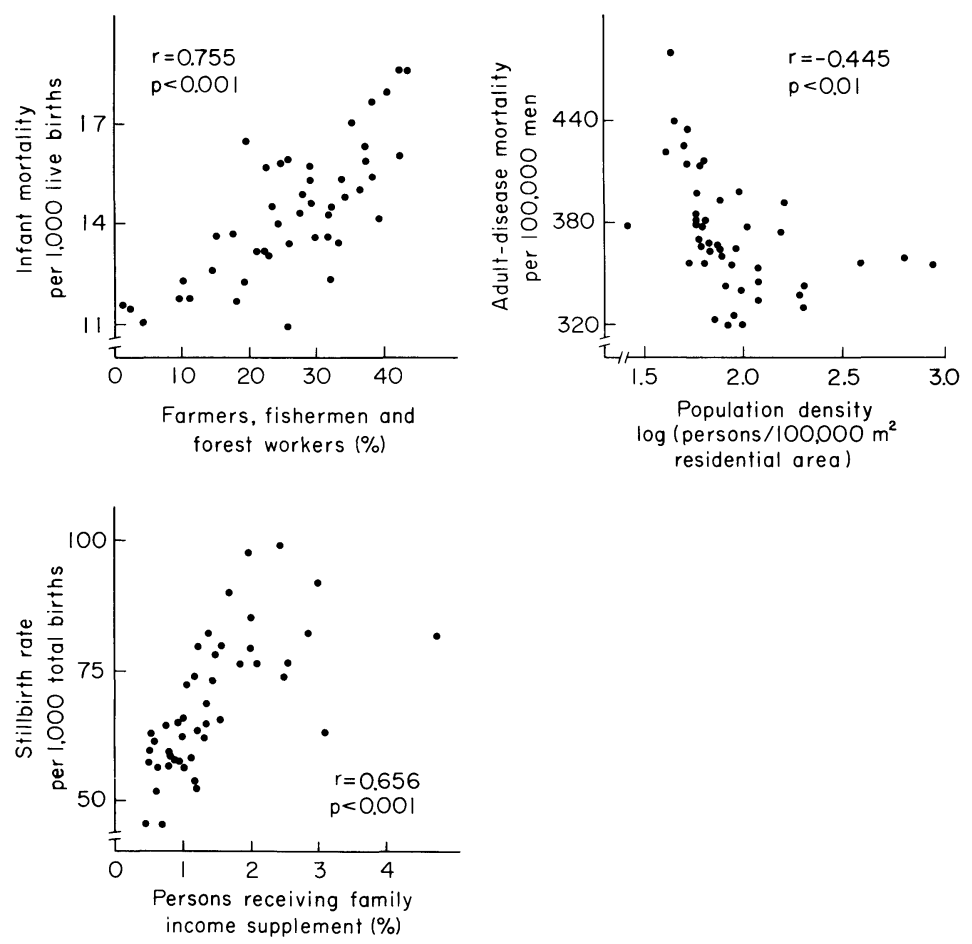

Fig. 2. Relationships between social life factors and infant and adult-disease mortality and stillbirth rates in 46 prefectures in 1970. Farmers, fishermen and forest workers, population density and persons receiving family income supplement represent rural residence, urban residence and low income factors, respectively.

5) in 1970 and 1975 ; the rate was inversely related to urban residence factor (Indicator 16) in 1975. Fig. 2 exemplifies the effects of these factors on infant and adult-disease mortality and stillbirth rates. All these rates significantly decreased between 1970 and 1975 (Table 3).

\section{Discussion}

The results indicate that rural residence is an essential mortality factor affecting not only adults but also infants (and stillbirths) in Japan. The effects of rural residence on these mortality rates agree with our previous findings on the mortality of total male and female populations and on occupational and suicide mortality in Japan (Araki and Murata 1984a, b, 1985, 1986a, b). Thus, a remarkable international difference in the mortality effects of urbanisation is suggested.

Infant mortality is an excellent indicator of living conditions in areas; in Great Britain, the mortality is lower in rural areas with good living conditions, but where there are no specialised paediatric services on the scale of those in large cities (Meredith Davies 1983). In Japan, living conditions are not considered to be better in rural than in urban areas; economic, educational, cultural and 
hygienic factors, e.g., savings of household, revenue of prefecture, higher education, newspaper circulation and domestic water supply, are inversely associated with rural residence (Fig. 1). Therefore, living conditions might be the major differential in the mortality effects of urban and rural residence.

Low income factor was significantly related to stillbirth rate in this study. This finding is compatible with the recent observation that artificial abortion due to economic reasons was responsible for 14 percent of all causes of stillbirth in 1983 in Japan (Health and Welfare Statistics Association 1985). Low income factor was also positively related to the mortality of total male population and skilled and manufacturing trades workers and to suicide mortality in middle-aged men in Japan (Araki and Murata 1985, 1986a, b). These findings are consistent with the observations in Great Britain, in which stillbirth and perinatal mortality rates and the rates from most of the major causes of death increase from social class I to V (McKeown and Lowe 1974 ; Meredith Davies 1983).

Finally, old age group factor was inversely related to adult-disease mortality in men. This finding coincides with our previous report, in which old age group factor was both positively and inversely related to all-causes mortality in men; young age group factor was positively related to the mortality (Araki and Murata 1986b). Further study is needed to investigate the effects of these factors on age-specific mortality rate. Similarly, sociological study is necessary to explain the inverse effects of population growth by social mobility on stillbirth rate.

\section{References}

1) Araki, S. \& Murata, K. (1984a) Suicide mortality in Japan: Changes during the past 15 years and the effects of social and demographic factors. Jap. J. publ. Hlth, 31, 651-657. (Japanese with English abstract)

2) Araki, S. \& Murata, K. (1984b) Social life factors affecting suicide in Japan. In : Proceedings of 43rd Annual Meeting of Japanese Society of Public Health, I, edited by Japanese Society of Public Health, Osaka Association of Public Health, Osaka, p. 533. (Japanese)

3) Araki, S. \& Murata, K. (1985) Mortality of working and non-working populations : Effects of social life factors. J. human Ergol., 14, 89-98.

4) Araki, S. \& Murata, K. (1986a) Factors affecting suicide mortality in young, middleaged and elderly men. J. biosoc. Sci., 18, 103-108.

5) Araki, S. \& Murata, K. (1986b) Social life factors affecting the mortality of total Japanese population. Jap. J. publ. Hlth, 33, Suppl. in press.

6) Japan Ministry of Health and Welfare (1974) Special Report of Vital Statistics in 1970 : Cause-Specific Age-Adjusted Mortality. Health and Welfare Statistics Association, Tokyo. (Japanese)

7) Japan Ministry of Health and Welfare (1978) Special Report of Vital Statistics in 1975 : Cause-Specific Age-Adjusted Mortality. Health and Welfare Statistics Association, Tokyo. (Japanese)

8) Japan Ministry of Health and Welfare (1984) Vital Statistics 1982 Japan. Health and Welfare Statistics Association, Tokyo.

9) Japan Prime Minister's Office (1977) System of Statistical Indicators on Life. Japan Statistical Association, Tokyo. (Japanese) 
10) Health and Welfare Statistics Association (1985) Trends in National Health. Health and Welfare Statistics Association, Tokyo, pp. 67-70. (Japanese)

11) McKeown, T. \& Lowe, C.R. (1974) An Introduction to Social Medicine. 2nd ed., Blackwell, Oxford, pp. 59-72.

12) Meredith Davies, J.B. (1983) Community Health, Preventive Medicine and Social Services. 5th ed., Bailliere Tindall, London, pp. 48-59.

13) Sauer, H.I. (1980) Geographic Patterns in the Risk of Dying and Associated Factors Ages 35-74 Years (Vital Health Statistics Series 3 Analytical Studies No. 18). US National Center for Health Statistics, Rockville. 